Research Paper

\title{
The Impact of Primary Tumor Sidedness on the Effect of Regorafenib in Refractory Metastatic Colorectal Cancer
}

\author{
Sang Eun Yoon, Su Jin Lee, Jeeyun Lee, Se Hoon Park, Joon Oh Park, Ho Yeong Lim, Won Ki Kang, Young \\ Suk Park, Seung Tae Kim ${ }^{\bowtie}$ \\ Division of Hematology-Oncology, Department of Medicine, Samsung Medical Center, Sungkyunkwan University School of Medicine, Seoul, Korea \\ $\square$ Corresponding author: Seung Tae Kim, M.D., Ph.D., Division of Hematology/Oncology, Department of Medicine, Samsung Medical Center, Sungkyunkwan \\ University School of Medicine, 81 Irwon-ro, Gangnam-gu, Seoul 06351, Korea. Tel.: +82-2-3410-0297, Fax: +82-2-3410-1754, Email: seungtae1.kim@samsung.com \\ (c) Ivyspring International Publisher. This is an open access article distributed under the terms of the Creative Commons Attribution (CC BY-NC) license \\ (https://creativecommons.org/licenses/by-nc/4.0/). See http://ivyspring.com/terms for full terms and conditions.
}

Received: 2018.08.09; Accepted: 2019.01.17; Published: 2019.03.03

\begin{abstract}
Recently, the sidedness of the primary tumor (right versus left) has been investigated for its ability to prognosticate and predict outcomes. We evaluated the effect of regorafenib based on KRAS mutation status and the sidedness of the primary tumor in patients with metastatic colorectal cancer (mCRC). We analyzed 135 patients with refractory metastatic colorectal cancer (mCRC) being treated with regorafenib at Samsung Medical Center, between January 2014 and January 2018. Primary tumors originating in the splenic flexure, descending colon, sigmoid colon, rectum, or proximal third of the transverse colon were defined as left-sided CRC (LC). Primary tumors originating in the appendix, cecum, ascending colon, hepatic flexure, or distal two-thirds of the transverse colon were defined as right-sided CRC (RC). Among all 135 patients, 100 (74.1\%) had left sided colon cancer and 35 (25.9\%) had right-sided colon cancer. No patients achieved a complete response, but four achieved a partial response, revealing a response rate (RR) of $3.0 \%$. Thirty-seven patients had stable disease, yielding a disease control rate (DCR) of $30.4 \%$. There was no difference in RR or DCR according to the location of the primary tumor (LC vs. RC). A significant difference in progression free survival (PFS) with regorafenib was observed between the LC and RC groups (2.6 months; $95 \% \mathrm{Cl}, 2.0$ to 3.1 vs. 1.9 months; $95 \% \mathrm{Cl}, 1.6$ to $2.3 ; P=0.04$, respectively). In a subpopulation with wild type KRAS, PFS with regorafenib was also significantly different between the LC and RC groups ( 2.9 months; $95 \% \mathrm{Cl}, 1.5$ to 4.3 vs. 2.1 months; $95 \% \mathrm{Cl}, 0.6$ to $3.6 ; P=0.04$ ). On multivariate analysis, the sidedness of the primary tumor (LC vs. RC) and the number of metastatic sites $(\leq 1$ vs. $2>)$ had a prognostic effect on PFS $(P=0.01$ and $P=0.01$, respectively). Regorafenib is a current standard treatment for CRC, but treatment outcomes may be improved if regorafenib is administered based on the appropriate biomarker.
\end{abstract}

Key words: sidedness, KRAS, regorafenib, colorectal cancer

\section{Introduction}

The prevalence of colorectal cancer (CRC) is gradually increasing, and the mortality of metastatic CRC (mCRC) is also increasing in worldwide [1]. The treatment for mCRC is systemic chemotherapy including 5-fluoropyrimidines (5FU), oxaliplatin, irinotecan and molecularly targeted agents such as anti-VEGF and anti-EGFR inhibitors [2-4]. However, a large number of patients still experience disease progression after treatment with available chemotherapies.

Regorafenib is a novel oral multi-kinase inhibitor that blocks the activity of several protein kinases, including kinases involved in the regulation of tumor angiogenesis, oncogenesis, and the tumor microenvironment [5]. Regorafenib has roles in wide ranges of angiogenic factors, the tumor microenvironment and oncogenic kinase. A novel phase III trial (CORRECT) announced that further treatment with regorafenib is acceptable for patients with mCRC that is refractory to standard chemotherapies. The CORRECT trial demonstrated an improvement in overall survival 
(OS) among regorafenib-treated patients in comparison to those randomized to receive best supportive care after disease progression while on standard therapy (6.4 versus 5.9 months, respectively) [6]. Another phase III trial in Asian patients with refractory $\mathrm{mCRC}$ also reported improved OS with regorafenib compared to placebo. These findings have provided evidence for regorafenib to be used as a new line of therapy in refractory $\mathrm{mCRC}$ patients [7]. However, the identification and confirmation of novel biomarkers is required so that regorafenib can be selectively targeted to responsive $\mathrm{mCRC}$ patients.

Assessing the sidedness of primary tumors is a novel theme in the study of colorectal cancer. There are many differences in molecular features between left and right-sided colon cancer. Recently, the sidedness of the primary tumor (right versus left side) has been investigated for its role in helping prognosticate and predict outcomes. The CRYSTAL and FIRE-3 studies examined the potential prognostic and predictive value of primary tumor location in mCRC patients with wild type KRAS being treated with FOLFIRI alone or in combination with cetuximab or bevacizumab [8, 9]. In patients treated with bevacizumab-containing chemotherapy, there was no difference in PFS based on the primary tumor location. Tumor location within colon cancer is known as a strong predictor of PFS with cetuximab therapy $[10,11]$.

Herein, we evaluated the effect of regorafenib according to KRAS mutation status and the sidedness of the primary tumor in $\mathrm{mCRC}$ patients.

\section{Materials and Methods}

\section{Patients}

We analyzed 135 refractory mCRC patients treated with regorafenib at Samsung Medical Center, Seoul, Korea, between January 2014 and January 2018. All patients had previously received fluoropyrimidines (FU), oxaliplatin, and irinotecan with biologic agents like cetuximab or bevacizumab. The clinical and pathological records were analyzed respectively. The following clinical data were gathered: age, gender, disease status, primary colon cancer site, Kirsten-ras (KRAS) mutational status and information on chemotherapy, performance status and survival. The left side of the colon was defined as the proximal third of the transverse colon, splenic flexure, descending colon, sigmoid colon, and rectum and the right side of the colon was defined as the cecum, ascending colon, hepatic flexure and distal two-thirds of the transverse colon $[8,12]$.

\section{Regorafenib}

Patients received oral regorafenib $160 \mathrm{mg}$ once daily for the first three weeks of each four-week cycle until disease progression, death, or unacceptable toxic effects. Generally, patients were followed up every week during the first two cycles, then every two weeks thereafter. All tumor measurements were assessed after every two cycles of chemotherapy, using computed tomography and other tests that were used initially in tumor staging.

\section{Statistical Methods}

Descriptive statistics were reported as proportions and medians. Treatment outcomes were response rate (RR) and progression-free survival (PFS). The response assessment was defined as complete response $(\mathrm{CR})$, partial response $(\mathrm{PR})$, stable disease (SD) and progressive disease (PD), based on the RECIST criteria (version 1.1) [13]. PFS was defined as the time from the initiation of bevacizumab-based chemotherapy to the date of disease progression or death from any cause. PFS was estimated using the Kaplan-Meier method with log-rank analysis. A two-sided p-value of less than 0.05 was considered statistically significant. The X2-test or Fisher's exact test was used to compare categorical variables. Two-sided null hypotheses of no difference were rejected if p-values were less than 0.05 , or equivalently, if the $95 \%$ confidence intervals (CIs) of risk point estimates excluded 1. Cox proportional hazards regression modeling was employed in univariate analysis to identify the significant, independent, prognostic factors of various clinical parameters for survival. All analyses were performed using SPSS version 19.0 (SPSS Inc., Chicago, IL, USA).

\section{Results}

\section{Patients}

The baseline characteristics of the 135 patients based on the sidedness of the primary tumor are shown in Table 1. Among the 135 patients, 100 (74.1\%) had LC, and 35 (25.9\%) had RC. KRAS mutation status was available in 120 patients (88.9\%), and MSI status was available in 71 patients $(52.6 \%)$. Patient characteristics were generally similar between RC and LC groups.

\section{Treatment efficacy}

No patients achieved CR, yet four achieved PR, yielding a RR of $3.0 \%$. Thirty-seven patients had SD, producing a disease control rate (DCR) of 30.4\% (Table 2). There was no difference in RR or DCR based on the location of the primary tumor ( $\mathrm{LC}$ vs. RC). The median PFS for regorafenib was 2.4 months $(95 \% \mathrm{CI}$, 2.0 to 2.8) (Figure 1A). A significant difference in PFS was observed between LC and RC groups (2.6 months; $95 \%$ CI, 2.0 to 3.1 vs. 1.9 months; $95 \%$ CI, 1.6 
to 2.3; $P=0.04$, respectively) (Figure $1 \mathrm{~B}$ ). There was no observable difference in PFS according to KRAS status (Figure 2A). In a subpopulation with a KRAS mutation, there was no significant difference in PFS with regorafenib between the LC and RC groups (2.0 months; $95 \%$ CI, 1.5 to 2.5 vs. 1.9 months; $95 \%$ CI, 1.5 to 2.0; $P=0.75$ ) (Figure 2B). However, in a subpopulation with wild type KRAS, PFS with regorafenib was significantly different between the LC and RC groups (2.9 months; $95 \%$ CI, 1.5 to 4.3 vs. 2.1 months; $95 \% \mathrm{CI}, 0.6$ to $3.6 ; P=0.04$ ) (Figure $2 \mathrm{C}$ ).

\section{Prognostic analysis}

Prognostic analyses for PFS with regorafenib are displayed in Table 3. In univariate analysis, the sidedness of the primary tumor (LC vs. RC) and the number of metastatic sites ( $\leq 1 \mathrm{vs} .2>$ ) had a prognostic effect on PFS. In multivariate analysis, these two factors still had a prognostic effect on PFS (sidedness of primary tumor, hazard ratio [HR], $1.71 ; 95 \% \mathrm{CI}$, 1.13 to 2.59; $P=0.01$, number of metastatic sites, HR, $1.71 ; 95 \% \mathrm{CI}, 1.13$ to $2.57 ; P=0.01$, respectively).

\section{Discussion}

The current study sought to investigate treatment outcomes of regorafenib according to the sidedness of the primary tumor and the KRAS mutation status in refractory mCRC patients. This analysis revealed that LC group had better PFS than RC (2.6 months va.1.9 months, $\mathrm{p}=0.04)$. In a subpopulation with wild type KRAS, PFS with regorafenib was also significantly different between the LC and RC groups (2.9 months, vs. 2.1 months; $\mathrm{P}=0.04$ ).
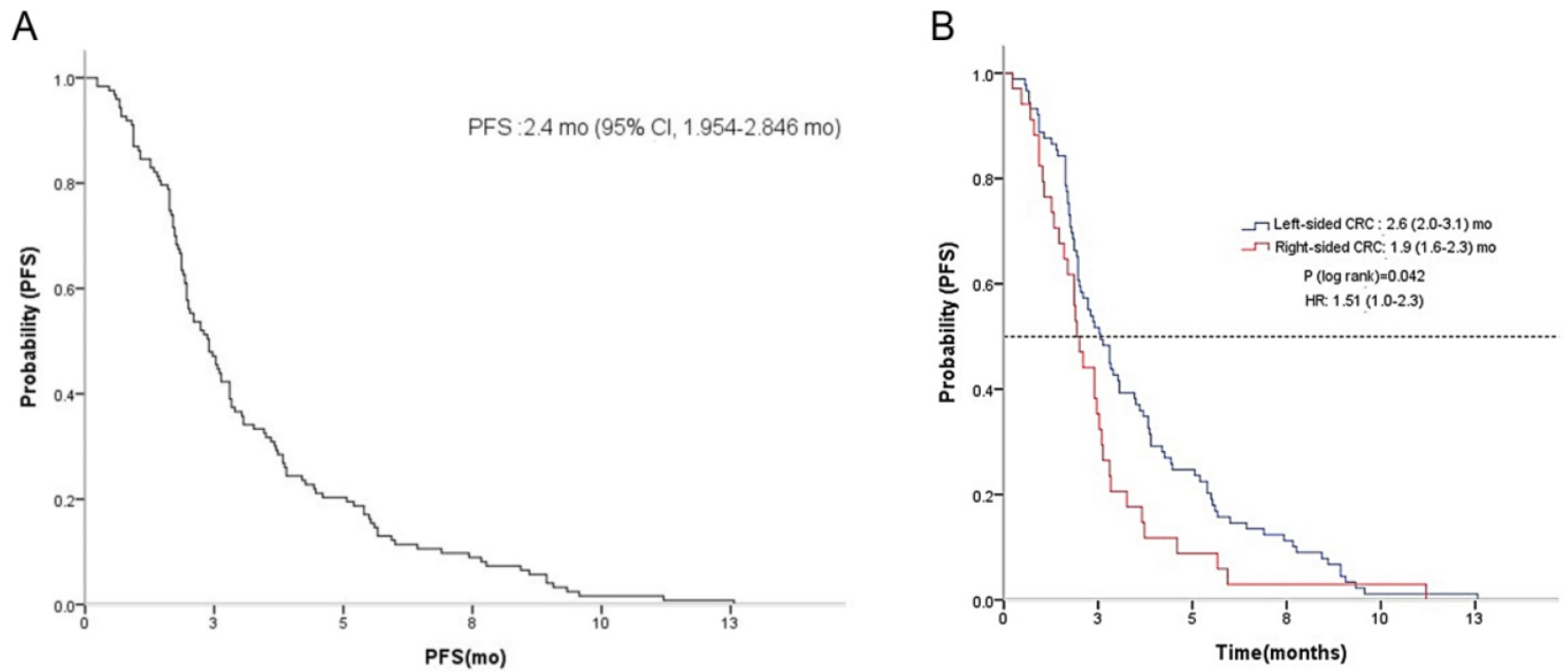

Figure 1. Kaplan-Meier estimate of progression-free survival (PFS) in mCRC patients with regorafenib $\mathbf{A}$ and between LC and RC groups $\mathbf{B}$.

A

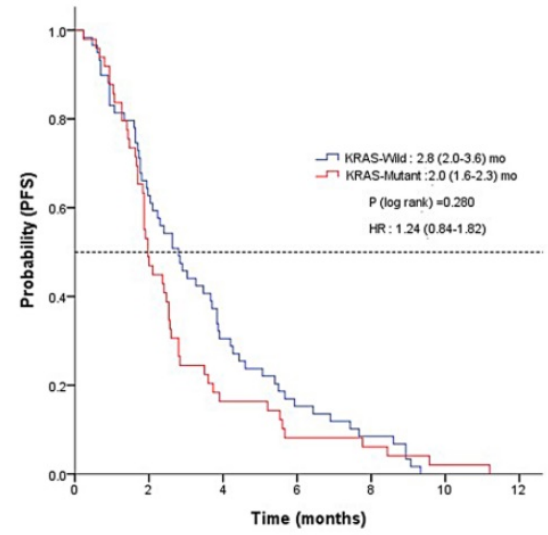

B

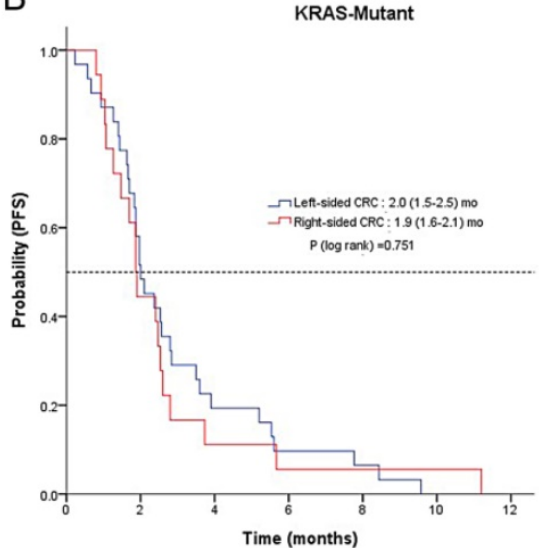

C
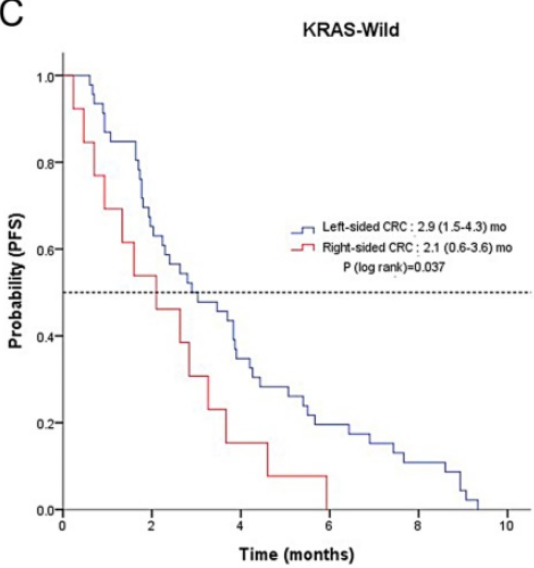

Figure 2. Kaplan-Meier analysis of PFS according to KRAS status A, tumor-sidedness in mutant type KRAS patients B and in wild KRAS patients $\mathbf{C}$. 
Table 1. Patient characteristics according to tumor sidedness $(\mathrm{N}=135)$

\begin{tabular}{|c|c|c|c|}
\hline & $\begin{array}{l}\text { Left sided CRC, } \\
\text { N }(\%)\end{array}$ & $\begin{array}{l}\text { Right sided CRC, } \\
\text { N (\%) }\end{array}$ & p-value ${ }^{\dagger}$ \\
\hline Patient Number & $100(100.0 \%)$ & $35(100.0 \%)$ & \\
\hline Age & & & 1.000 \\
\hline$<65$ & $83(83.0 \%)$ & $29(82.9 \%)$ & \\
\hline$\geq 65$ & $17(17.0 \%)$ & $6(17.1 \%)$ & \\
\hline Sex & & & 0.558 \\
\hline Men & $55(55.0 \%)$ & $17(48.6 \%)$ & \\
\hline Women & $45(45.0 \%)$ & $18(51.4 \%)$ & \\
\hline ECOG & & & 0.335 \\
\hline 0 & $6(6.0 \%)$ & $4(11.4 \%)$ & \\
\hline $1-2$ & $94(94.0 \%)$ & $31(88.6 \%)$ & \\
\hline Disease status & & & 1.000 \\
\hline Metastatic & $67(67.0 \%)$ & $24(68.6 \%)$ & \\
\hline Recurrent & $33(33.0 \%)$ & $11(31.4 \%)$ & \\
\hline KRAS mutation & & & 0.146 \\
\hline Wild & $53(53.0 \%)$ & $14(40.0 \%)$ & \\
\hline Mutant & $35(35.0 \%)$ & $18(51.4 \%)$ & \\
\hline Unknown & $12(12.0 \%)$ & $3(8.6 \%)$ & \\
\hline \multicolumn{2}{|c|}{$\begin{array}{l}\text { Number of previous systemic anticancer } \\
\text { therapies for metastatic disease }\end{array}$} & & 0.326 \\
\hline$\leq 3$ & $46(46.0 \%)$ & $20(57.2 \%)$ & \\
\hline$>3$ & $54(54.0 \%)$ & $15(42.9 \%)$ & \\
\hline Metastatic site & & & 0.828 \\
\hline $1-2$ & $71(71.0 \%)$ & $26(74.3 \%)$ & \\
\hline$\geq 3$ & $29(29.0 \%)$ & $9(25.7 \%)$ & \\
\hline
\end{tabular}

Table 2. Best overall response rate (RR) and disease control rate $(D C R)$ in patients receiving regorafenib

\begin{tabular}{lclll}
\hline & $\begin{array}{l}\text { Total } \\
\text { N (\%) }\end{array}$ & $\begin{array}{l}\text { LC } \\
\text { N (\%) }\end{array}$ & $\begin{array}{l}\text { RC } \\
\text { N (\%) }\end{array}$ & p-value $^{*}$ \\
\hline $\begin{array}{l}\text { Response rate } \\
\text { (above PR) }\end{array}$ & $4(3.0 \%)$ & $4(4.0 \%)$ & $0(0.0 \%)$ & 0.573 \\
$\begin{array}{l}\text { Disease control rate } \\
\text { (above SD) }\end{array}$ & $41(30.4 \%)$ & $34(34.0 \%)$ & $7(20.0 \%)$ & 0.092 \\
PR & $4(3.0 \%)$ & $4(4.0 \%)$ & $0(0.0 \%)$ & \\
SD & $37(27.4 \%)$ & $30(30.0 \%)$ & $7(20.0 \%)$ & \\
PD & $90(66.7 \%)$ & $62(62.0 \%)$ & $28(80.0 \%)$ & \\
Not evaluable & $4(3.0 \%)$ & $4(4.0 \%)$ & $0(0.0 \%)$ & \\
\hline * p value is calculated by chi-square test. &
\end{tabular}

A number of differences have been established between RC and LC. RCs are more likely to be exophytic, diploid, mucinous in histology, predominantly MSI-H and contain RAS/RAF mutants, whereas LCs are often infiltrating, aneuploid, present with obstructive symptoms, and have predominant chromosomal instability [14-16]. Recently, gene expression profiles showed that CRC subtypes were differently distributed between RC and LC. In LC, VEGR-VEGFR pathway and stromal pathway were activated more abundantly as compared to RC $[17,18]$. Tissue expression of VEGF-A has also been demonstrated to vary depending on the location of the primary tumor, with higher expression observed in tumors from the left side than in tumors on the right side. These finding suggested that anti-angiogenetic agents including regorafenib might be more potent in LC. Regorafenib non-specifically binds to several intracellular kinases with potent inhibitory activity against vascular endothelial growth factor receptors 1-3 (VEGFR1, VEGFR2, and VEGFR3), PDGFRB, FGFR1, RAF, and TIE2, and the mutant oncogenic kinases KIT, RET, and BRAF [19]. However, this concept needs the further investigation. Although this study showed that a significant difference in progression free survival (PFS) with regorafenib was observed between the LC and RC groups. This study has some limitations such as the retrospective natures, the small sample size, the lack of consistency in patients' follow-up and overall survival. Thus, findings in this study must be interpreted with caution.

Table 3. Univariate analyses of PFS.

\begin{tabular}{|c|c|c|c|c|c|}
\hline \multirow[b]{2}{*}{ Parameter } & \multirow[b]{2}{*}{ PFS } & \multicolumn{2}{|c|}{ Univariate analyses } & \multicolumn{2}{|c|}{ Multivariate analyses } \\
\hline & & $\begin{array}{l}\text { Adjusted HR } \\
(95 \% \mathrm{CI})\end{array}$ & $\begin{array}{l}\text { p- } \\
\text { value* }\end{array}$ & $\begin{array}{l}\text { Adjusted HR } \\
(95 \% \mathrm{CI})\end{array}$ & $\begin{array}{l}\text { p- } \\
\text { value* }\end{array}$ \\
\hline Age & & & 0.143 & & \\
\hline$\leq 70$ & 2.400 & 1.604 & & & \\
\hline$>70$ & 1.967 & $(0.852-3.018)$ & & & \\
\hline Sex & & & 0.814 & & \\
\hline Male & 2.800 & 1.044 & & & \\
\hline Female & 2.100 & $(0.729-1.496)$ & & & \\
\hline PS (ECOG) & & & 0.908 & & \\
\hline 0 & 1.933 & 0.960 & & & \\
\hline $1-2$ & 2.533 & $(0.480-1.919)$ & & & \\
\hline \multicolumn{2}{|c|}{ Primary tumor location } & & 0.042 & & 0.012 \\
\hline Left & 2.567 & 1.519 & & 1.709 & \\
\hline Right & 1.933 & $(1.016-2.270)$ & & $(1.127-2.592)$ & \\
\hline KRAS & & & 0.280 & & \\
\hline Wild & 2.800 & 1.237 & & & \\
\hline Mutant & 1.967 & $(0.841-1.822)$ & & & \\
\hline \multicolumn{2}{|c|}{ No. of Metastatic sites } & & 0.037 & & 0.011 \\
\hline$\leq 1$ & 2.533 & 1.523 & & 1.705 & \\
\hline$>2$ & 1.933 & $(1.025-2.261)$ & & $(1.132-2.566)$ & \\
\hline \multicolumn{3}{|c|}{ Previous anti-VEGF treatment } & 0.041 & & \\
\hline NO & 2.833 & 1.720 & & & \\
\hline YES & 2.233 & $(1.023-2.894)$ & & & \\
\hline \multicolumn{3}{|c|}{ Previous anti-EGFR treatment } & 0.605 & & \\
\hline NO & 2.367 & 0.908 & & & \\
\hline YES & 2.633 & $(0.630-1.309)$ & & & \\
\hline \multicolumn{3}{|c|}{$\begin{array}{l}\text { Number of previous systemic anticancer } \\
\text { therapies }\end{array}$} & 0.285 & & \\
\hline$\leq 3$ & 2.100 & 0.823 & & & \\
\hline $3>$ & 2.400 & $(0.576-1.176)$ & & & \\
\hline
\end{tabular}

* Univariate and multivariate analysis to identify the significant, independent, prognostic factors of various clinical parameters for survival is calculated by Cox proportional hazards regression model.

The present study revealed that the objective RR and the DCR were $3.0 \%$ and $30.4 \%$, respectively. These findings were consistent with previous phase III trials for regorafenib. We also analyzed the RR and DCR for regorafenib based on the sidedness of the primary tumor. Although there was a small sample size, there was no difference in RR or DCR between LC and RC. 
KRAS is a proto-oncogene encoding a small 21 $\mathrm{kD}$ guanosine triphosphate/guanosine diphosphate binding protein, and it is involved in regulating cellular responses to extracellular stimuli $[20,21]$. The mutation status of RAS is a novel biomarker for determining the use of anti-EGFR therapy [22, 23]. In the present study, we analyzed the relationship between the treatment outcome of regorafenib and the KRAS mutation status. The role of the mutational status of KRAS to effect for regorafenib is controversial. In CORRECT, there was no relation between KRAS mutation status and outcomes of regorafenib. However, in REBACCA, the mutational status of KRAS mutation was one of poor prognostic factors for survival. In current study, there was no significant difference in PFS according to the mutational status of KRAS. However, in subgroup analysis for patients with the only wild type of KRAS, PFS to regorafenib was also significantly different between the LC and $\mathrm{RC}$ groups. The sidedness of primary tumor and the mutational status of KRAS in CRC are regarded as novel biomarkers to both the prognosis and the prediction to molecularly targeted agents. However, there was few data for the relation to between the effect regorafenib and these biomarkers. More studies must be investigated in the future to confirm this concept.

In the period of the study, the national reimbursement program didn't cover the cost of testing NRAS and HRAS mutation. Thus, we can't evaluate the status of NRAS, and HRAS mutation. However, currently, this problem of the reimbursement program is resolved because the Korean reimbursement program started to cover the cost of next generation sequencing (NGS) including KRAS, HRAS, NRAS and BRAF from 2018.

$\mathrm{RC}$ and LC are now recognized to have distinct clinical and genomic features; however, treatment strategies considering molecular heterogeneity have not been established. Our analysis suggests that regorafenib a current standard treatment that may have improved treatment outcomes if given based on the appropriate biomarker.

\section{Competing Interests}

The authors have declared that no competing interest exists.

\section{References}

1. Torre LA, Bray F, Siegel RL, et al. Global cancer statistics, 2012. CA Cancer J Clin. 2015; 65: 87-108.

2. Riechelmann R, Grothey A. The role of regorafenib in metastatic colorectal cancer. Lancet Oncol. 2015; 16: 596-597.

3. Nielsen DL, Palshof JA, Larsen FO, et al. A systematic review of salvage therapy to patients with metastatic colorectal cancer previously treated with fluorouracil, oxaliplatin and irinotecan $+/$ - targeted therapy. Cancer Treat Rev. 2014; 40: 701-715.
4. Ciombor $\mathrm{KK}, \mathrm{Wu} \mathrm{C}$, Goldberg RM. Recent therapeutic advances in the treatment of colorectal cancer. Annu Rev Med. 2015; 66: 83-95.

5. Wilhelm SM, Dumas J, Adnane L, et al. Regorafenib (BAY 73-4506): a new oral multikinase inhibitor of angiogenic, stromal and oncogenic receptor tyrosine kinases with potent preclinical antitumor activity. Int J Cancer. 2011; 129: 245-255.

6. Grothey A, Van Cutsem E, Sobrero A, et al. Regorafenib monotherapy for previously treated metastatic colorectal cancer (CORRECT): an international, multicentre, randomised, placebo-controlled, phase 3 trial. Lancet. 2013; 381: 303-312.

7. $\mathrm{Li}$ J, Oin $\mathrm{S}, \mathrm{Xu} \mathrm{R}$, et al. Regorafenib plus best supportive care versus placebo plus best supportive care in Asian patients with previously treated metastatic colorectal cancer (CONCUR): a randomised, double-blind, placebo-controlled, phase 3 trial. Lancet Oncol. 2015; 16: 619-629.

8. Tejpar S, Stintzing S, Ciardiello F, et al. Prognostic and Predictive Relevance of Primary Tumor Location in Patients With RAS Wild-Type Metastatic Colorectal Cancer: Retrospective Analyses of the CRYSTAL and FIRE-3 Trials. JAMA Oncol. 2016; doi: 10.1001/jamaoncol.2016.3797.

9. Modest DP, Stintzing S, von Weikersthal LF, et al. Exploring the effect of primary tumor sidedness on therapeutic efficacy across treatment lines in patients with metastatic colorectal cancer: analysis of FIRE-3 (AIOKRK0306). Oncotarget. 2017; 8: 105749-105760.

10. Chen KH, Shao YY, Chen HM, et al. Primary tumor site is a useful predictor of cetuximab efficacy in the third-line or salvage treatment of KRAS wild-type (exon 2 non-mutant) metastatic colorectal cancer: a nationwide cohort study. BMC Cancer. 2016; 16: 327

11. Heinemann V, Modest DP, Fischer von Weikersthal L, et al. Gender and tumor location as predictors for efficacy: Influence on endpoints in first-line treatment with FOLFIRI in combination with cetuximab or bevacizumab in the AIO KRK 0306 (FIRE3) trial. J Clin Oncol. 2014; 32: 3600.

12. Holch JW, Ricard I, Stintzing S, et al. The relevance of primary tumour location in patients with metastatic colorectal cancer: A meta-analysis of first-line clinical trials. Eur J Cancer. 2017; 70: 87-98.

13. Eisenhauer EA, Therasse $P$, Bogaerts J, et al. New response evaluation criteria in solid tumours: revised RECIST guideline (version 1.1). Eur J Cancer. 2009. 45: 228-247.

14. Missiaglia E, Jacobs B, D'Ario G, et al. Distal and proximal colon cancers differ in terms of molecular, pathological, and clinical features. Ann Oncol. 2014; 25 : 1995-2001.

15. Iacopetta B. Are there two sides to colorectal cancer? Int J Cancer. 2002; 101: $403-408$

16. Azzoni C, Bottarelli L, Campanini N, et al. Distinct molecular patterns based on proximal and distal sporadic colorectal cancer: arguments for different mechanisms in the tumorigenesis. Int J Colorectal Dis. 2007; 22: 115-126.

17. Lee MS, Menter DG, Kopetz S. Right versus Left Colon Cancer Biology: Integrating the Consensus Molecular Subtypes. J Natl Compr Canc Netw 2017;15(3):411-419.

18. Guinney J, Dienstmann R, Wang X, et al. The consensus molecular subtypes of colorectal cancer. Nat Med: 2015;21(11):1350-1356.

19. Strumberg D, Scheulen ME, Schultheis B, et al. Regorafenib (BAY 73-4506) in advanced colorectal cancer: a phase I study. Br J Cancer. 2012; 106: 1722-1727.

20. Tan C, Du X. KRAS mutation testing in metastatic colorectal cancer. World J Gastroenterol. 2012; 18: 5171-5180.

21. Dinu D, Dobre M, Panaitescu E, et al. Prognostic significance of KRAS gene mutations in colorectal cancer--preliminary study. J Med Life. 2014; 7: 581-587.

22. von Einem JC, Heinemann V, von Weikersthal LF, et al. Left-sided primary tumors are associated with favorable prognosis in patients with KRAS codon 12/13 wild-type metastatic colorectal cancer treated with cetuximab plus chemotherapy: an analysis of the AIO KRK-0104 trial. J Cancer Res Clin Oncol. 2014; 140: 1607-1614

23. Brule SY, Jonker DJ, Karapetis CS, et al. Location of colon cancer (right-sided versus left-sided) as a prognostic factor and a predictor of benefit from cetuximab in NCIC CO.17. Eur J Cancer. 2015; 51: 1405-1414. 